\title{
Utilisation des services écosystémiques pour la définition de seuils de rejets des eaux usées industrielles. Vers un outil d'aide à la décision pour les entreprises.
}

Valérie Laforest, Sophie Grazilhon and Eric Piatyszek

\section{OpenEdition}

\section{Journals}

Electronic version

URL: http://journals.openedition.org/eue/1758

ISSN: 1916-4645

Publisher

Institut national de Recherche scientifique Urbanisation Culture et Société

\section{Electronic reference}

Valérie Laforest, Sophie Grazilhon and Eric Piatyszek, « Utilisation des services écosystémiques pour la définition de seuils de rejets des eaux usées industrielles. Vers un outil d'aide à la décision pour les entreprises. », Environnement Urbain / Urban Environment [Online], Volume 11 | 2017, Online since 07 July 2017, connection on 01 May 2019. URL : http://journals.openedition.org/eue/1758

This text was automatically generated on 1 May 2019.

(c) Institut National de Recherche Scientifique Centre Urbanisation Culture et Société 


\section{Utilisation des services} écosystémiques pour la définition de seuils de rejets des eaux usées industrielles. Vers un outil d'aide à la décision pour les entreprises.

Valérie Laforest, Sophie Grazilhon and Eric Piatyszek

\section{Contexte et problématique de la gestion des eaux usées industrielles}

1 L'industrie est une interface majeure entre l'humain et le milieu naturel. Elle prélève des ressources dans le milieu naturel afin de concevoir des biens ou des services qui répondent aux besoins de l'humain et rejette des déchets, des effluents dans ce milieu naturel. L'approche linéaire historique où l'utilisation de la ressource est faite de manière illimitée et la production de déchets est non gérée est en passe d'évoluer vers des dynamiques circulaires. En effet, des approches circulaires des flux émergent et font entrevoir des stratégies ayant pour objectifs la réduction des pressions de l'être humain sur son environnement. Les concepts soutenant ces approches s'appuient sur les synergies entre les cycles anthropiques et les cycles naturels. La symbiose entre ces deux éléments nécessite une approche aux interfaces entre l'être humain et le milieu naturel. (Orée, 2009 ; Grazilhon, 2015)

2 Un des enjeux des entreprises aujourd'hui est la gestion de l'eau qu'elles utilisent dans leur processus, et ce, pour faire face à la pression sur la préservation de la ressource.

3 De manière générale, la composition des eaux rejetées par l'humain diffère de celle d'un cours d'eau à l'état «naturel». De plus, les concentrations des substances, telles que la matière organique, certains métaux ou nutriments, sont plus élevées dans les eaux de rejets que dans le milieu naturel. En outre, les eaux de rejets peuvent également contenir 
des molécules ou composés créés par l'être humain (produits ou sous-produits chimiques) qui ne sont pas assimilables par les écosystèmes (Le Goff, 2004). Ainsi, au contact de ces effluents, les écosystèmes se modifient, puis se détériorent.

Pour évaluer l'état des écosystèmes aquatiques sur son territoire, l'Europe a mis en place une règlementation communautaire: la Directive Cadre sur l'Eau (DCE). Adoptée par le parlement européen le 23 octobre 2000, la DCE établit un cadre pour une politique communautaire dans le domaine de l'eau. En particulier, un des objectifs mis en avant par la DCE pour les états membres est l'atteinte d'ici 2015 du bon état écologique et chimique pour tous les cours d'eau. Pour chaque masse d'eau, un niveau de confiance est attribué, basé sur un état écologique et chimique. En 2009, en France, $45 \%$ des masses d'eau sont en bon état chimique, et $45 \%$ en «bon » ou " très bon » état écologique. En 2013, parmi les 11435 masses d'eau de surface (cours d'eau, plans d'eau, eaux de transition, eaux côtières) : 12,3 \% sont en très bon état écologique ; 31,1 \% sont en bon état écologique ; $41,5 \%$ sont en état écologique moyen ; 10,3\% sont en état écologique médiocre ; 3,8 \% sont en mauvais état écologique ; $1 \%$ sont en état indéterminé (les informations sont insuffisantes pour attribuer un état) (ONEMA, 2015). En 2015, seulement $53 \%$ des masses d'eau sont conformes aux objectifs de la Directive Cadre (EEA, 2015).

5 Ainsi, les rejets industriels, de par leur composition, peuvent impacter à la fois l'état écologique et l'état chimique des masses d'eau et donc rendre l'atteinte des objectifs de la DCE incertaine. Toutefois, la prise en compte des problématiques environnementales dans la gestion des émissions industrielles est aujourd'hui bien ancrée dans la règlementation française et européenne.

6 Ainsi, la directive européenne sur les émissions industrielles, dite IED (Industrial Emission Directive), impose aux états membres, et notamment à la France un cadre de fonctionnement afin de réduire les émissions industrielles dans leur ensemble (Parlement européen et conseil de l'Union Européenne, 2010).

7 Les installations soumises à la directive IED sont contraintes à :

- Faire une demande d'autorisation à exploiter,

- identifier et mettre en place des techniques de production ou de traitement des émissions (air, eau) ou des déchets ayant des performances au moins équivalentes à celles des Meilleures Techniques Disponibles (MTD) afin d'éviter certaines émissions,

- prendre en compte les contraintes locales (qui fait notamment référence à la DCE) pour l'élaboration des Valeurs Limites d'Emission (VLE).

8 La transcription en droit français de la directive IED repose, en partie, sur la réglementation des Installations Classées pour le Protection de l'Environnement (ICPE) pour laquelle une procédure d'autorisation existe depuis 1977. Pour les ICPE, leurs émissions sont règlementées de manière générale en termes d'émissions par l'arrêté du 2 février 1998. Pour la plupart des secteurs d'activités, des arrêtés sectoriels plus précis fixent certaines conditions d'exploitations, et certaines valeurs d'émissions à ne pas dépasser. L'élaboration des seuils d'émissions se fonde sur la règlementation d'une part dite « industrielle »: ICPE et IED, et, d'autre part, dite «milieu naturel » qui concerne notamment la protection de la ressource en eau : la DCE. La procédure d'autorisation, qui concerne les installations les plus susceptibles d'avoir des impacts sur les milieux aquatiques permet, la mise en application des objectifs réglementaires et en théorie l'articulation avec la DCE ainsi que les différents acteurs impliqués. 
9 Les projets soumis à autorisation selon la directive IED ou la règlementation ICPE suivent approximativement le même processus : une phase d'étude, pendant laquelle le projet prend sa forme définitive et où sont réalisées les études d'impact environnemental. Lorsque des eaux usées sont générées, cette phase d'étude permet de définir par rapport au milieu récepteur et à la règlementation des objectifs de traitement sur lesquels seront basé en partie le choix des techniques de traitement à mettre en place et un cahier des charges pour dimensionner les installations. Cette phase d'étude aboutit ainsi à l'élaboration d'un dossier de demande d'autorisation d'exploiter (DDAE), qui doit contenir différents éléments, dont l'étude d'impact. Ce dossier est transmis à la préfecture du département où le projet doit s'implanter pour la procédure complète liée à l'autorisation. Si le projet est autorisé, l'arrêté préfectoral d'autorisation d'exploiter fixe les conditions d'exploitation et en particulier les Valeurs Limites d'Emission (VLE) pour les effluents aqueux du projet. Une fois seulement le projet autorisé, il peut entrer en phase de réalisation puis d'exploitation.

Le processus de décision implique plusieurs catégories d'acteurs ayant un positionnement et une force différents dans les processus décisionnels (tableau 1). Grazilhon (2015) a montré dans sa thèse qu'il existe un déséquilibre entre les acteurs dans le processus décisionnel: deux acteurs forts (l'industriel et le préfet de département délivrant l'autorisation) cohabitent avec des acteurs faibles, notamment les acteurs représentant les milieux naturels.

Tableau 1 : Catégories d'acteurs impliqués dans les processus décisionnels relatifs à l'étude d'impacts

\begin{tabular}{|c|c|c|}
\hline $\begin{array}{l}\text { Acteurs « responsables " } \\
\text { acteurs forts }\end{array}$ & $\begin{array}{l}\text { acteurs « informés " } \\
\text { acteurs faibles }\end{array}$ & Acteurs absents \\
\hline $\begin{array}{l}\text { - les porteurs de projets / } \\
\text { entreprises } \\
\text { pétitionnaires, } \\
\text { - les préfets de } \\
\text { département. }\end{array}$ & $\begin{array}{l}\text { - Les centres techniques, } \\
\text { - L'ONEMA, } \\
\text { - Les agences de l'eau, } \\
\text { - La DREAL, } \\
\text { - Les administrations et } \\
\text { établissements publics, } \\
\text { - Les services } \\
\text { administratifs } \\
\text { particuliers, } \\
\text { - Les collectivités locales, } \\
\text { - Les associations, } \\
\text { - Le public. }\end{array}$ & $\begin{array}{l}\text { - Ecosystèmes } \\
\text { - pouvant être représentés par } \\
\text { les associations de } \\
\text { protection de la nature }\end{array}$ \\
\hline
\end{tabular}

11 Ce contexte réglementaire, qui à priori met la protection du milieu naturel au cœur des réflexions, ne permet pas, aujourd'hui, de le prendre en considération dans les processus décisionnels. Ainsi, la question posée est « comment prendre en considération le milieu récepteur comme acteur du système dans les processus décisionnels pour la gestion des rejets industriels?».

Pour répondre à cette question, cet article présente, dans la seconde partie, les éléments qui ont permis de mettre les services écosystémiques au cœur de la réflexion. La partie 3 , présentation de l'intégration des services écosystémiques dans la méthodologie proposée 
qui sera appliquée dans la partie 4. Une discussion terminera cet article en partie 5 avant la conclusion finale.

\section{Réflexions/concepts}

$$
\begin{aligned}
& \text { potentiellement un impact sur l'environnement } \\
& \text { L'idée ici est de réintégrer le milieu récepteur comme acteur } \\
& \text { rejet de produits aqueux. Pour cela il est proposé d'aborder } \\
& \text { effluents au travers de : } \\
& \text { - l'application des principes directeurs de l'ISO 9000, } \\
& \text { - la considération d'un effluent industriel comme un produit, } \\
& \text { - la prise en compte du milieu naturel comme client. }
\end{aligned}
$$
par leur nature, et représentés par des acteurs «faibles », c'est-à-dire, qui ne possèdent pas de pouvoir décisionnel dans les processus d'autorisation de projets ayant

L'idée ici est de réintégrer le milieu récepteur comme acteur du système en lien avec le rejet de produits aqueux. Pour cela il est proposé d'aborder la gestion de la qualité des

\subsection{Intégrer le milieu récepteur comme acteur du système industriel au travers la notion de qualité industrielle}

Le terme de qualité est fréquemment employé aujourd'hui dans divers domaines et disciplines. Afin de guider les entreprises dans le management de la qualité, en 1987 apparait la série des normes ISO 9000.

La norme ISO 9000 sur les Systèmes de management de la qualité s'intéresse particulièrement à la gestion de la qualité des produits entre les différentes parties prenantes, du sous-traitant au fournisseur et du fournisseur au client (figure 1). Ainsi, dans un contexte industriel, la norme ISO 9000 a été élaborée avec pour objectif d'aider " les organismes de tous types et de toutes tailles à mettre en cuvre et à appliquer des systèmes de management de la qualité efficaces » (ISO, 2005).

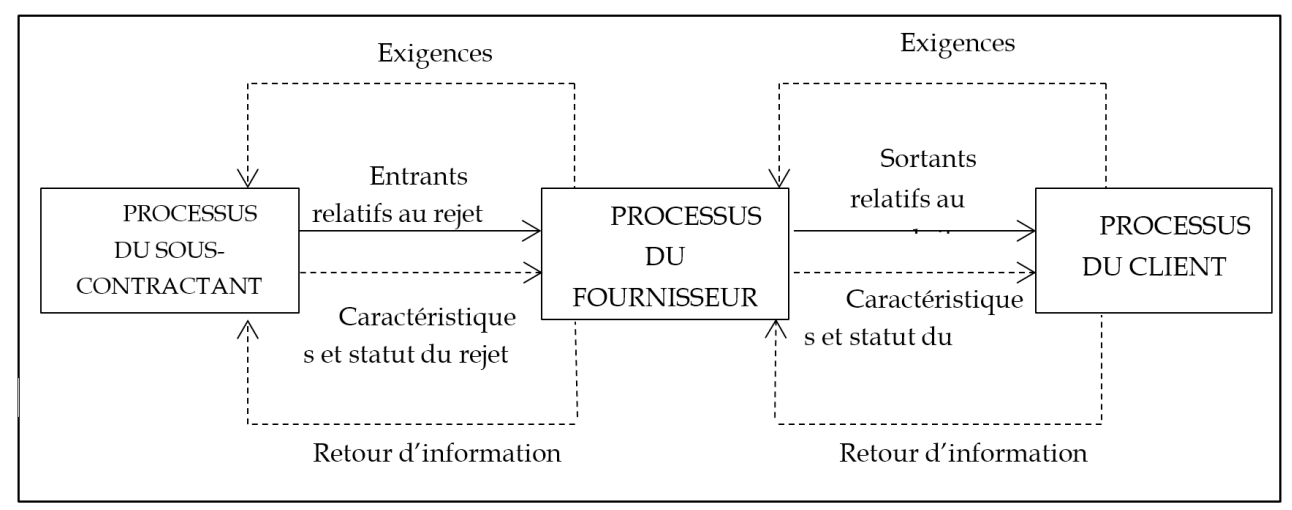

Figure 1 : Axes d'orientation de la démarche de management de la qualité (ISO, 2005)

Comme nous pouvons le constater sur la Figure 1, l'activité humaine est totalement isolée et déconnectée de son environnement ; l'environnement n'est pas pris en considération dans le processus de qualité.

Cependant, l'environnement est une composante essentielle du territoire (Cerceau, 2013) et doit donc s'inscrire dans les processus d'échange et de management des activités anthropiques sur ce territoire. De ce fait, le positionnement de l'environnement dans ces 
relations entre acteurs forts (Sébastien, 2011) doit être repensé non pas comme un élément qui supporte seulement des activités, mais comme acteur du système territorial sur lequel l'activité est implantée. En s'inscrivant dans ce schéma, l'environnement se positionne en relation directe avec le système fournisseur et donc indirectement avec le client. Le fournisseur étant l'activité anthropique sur laquelle l'étude est centrée. La figure 2 montre l'intégration des échanges fournisseur/environnement sur le même plan que les relations fournisseur/client (pour des raisons de lisibilité, les relations fournisseur/sous-traitant n'ont pas été représentées sur le schéma).

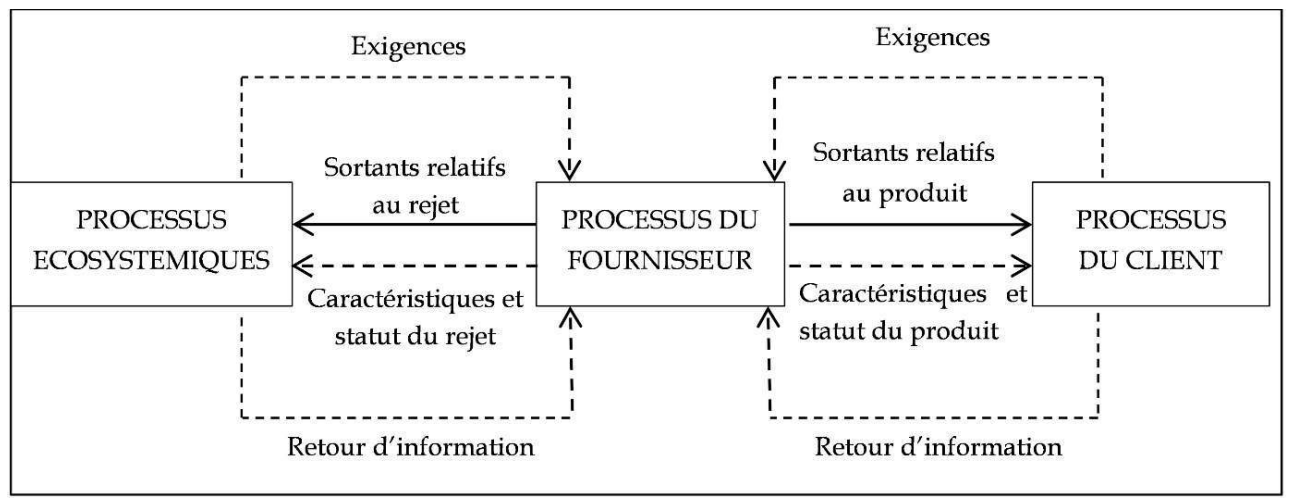

Figure 2: Proposition d'application du concept de qualité défini par l'ISO 9000 (ISO, 2005) aux échanges avec l'environnement

L'intégration de l'environnement dans le processus qualité nécessite néanmoins de considérer l'écosystème comme «client » du produit « rejet » qui est dans notre cas des effluents aqueux.

Or, la norme ISO 9000 (ISO, 2005) définit le client comme l'organisme ou la personne qui reçoit un produit ; un organisme étant un ensemble d'installations et de personnes avec des responsabilités, pouvoirs et relations (par exemple une entreprise, une institution, une association, etc.).

Dans le cas des rejets industriels aqueux, le client (le milieu récepteur pour un effluent) est donc défini comme l'ensemble des systèmes et des écosystèmes en contact avec ledit effluent et susceptibles d'être impactés par lui.

Par contre, la difficulté de prendre en compte un écosystème comme client ou de manière plus générale comme partie prenante est liée à son incapacité à exprimer un besoin (« les exigences » sur la figure 2). Cela nous amène à poser la question : «quels sont alors les besoins d'un écosystème et comment les évaluer?».

\subsection{Appréhension des besoins d'un écosystème}

Pour un organisme vivant, les besoins sont par définition les besoins élémentaires et biophysiologiques (Maslow, 1943), illustré par exemple dans (Rishi et al., 2007) (Noltemeyer et al., 2012). Ces besoins sont résumés par le concept d'homéostasie, développé par Bradford Cannon en 1932. L'homéostasie est un équilibre dynamique obtenu grâce à un ensemble de processus de régulation qui permettent la pérennité d'un système quelconque (vivant ou non, fermé ou ouvert) en dépit des contraintes extérieures (Cannon, 1932 ; Larousse, 2014). Notons que cette notion est étendue au domaine du nonvivant. Ainsi les besoins d'un milieu naturel se définissent aussi bien en termes d'état (qualité de l'eau, biodiversité, morphologie et hydraulique) que de mécanismes de 
régulation internes à l'écosystème qui lui permettent de conserver un état quasi stationnaire. La notion de besoin est ainsi appréhendée au travers de l'homéostasie de l'écosystème, c'est-à-dire de la composition de l'eau et des mécanismes de régulation, couramment appelés mécanismes d'autoépuration.

L'autoépuration est un ensemble de mécanismes visant à restaurer le milieu dans son état initial suite à une modification physique, chimique, et/ou biologique du milieu (Vagnetti et al. 2003). Ce processus a été mis en évidence et étudié dès les années 1970 (Knowles, Wakeford, 1978).

Afin de pouvoir évaluer les besoins du client Milieu naturel, il est donc nécessaire de trouver des outils permettant de quantifier l'autoépuration. L'évaluation de l'autoépuration dans un cours d'eau peut se faire de deux manières : expérimentale ou théorique.

L'approche expérimentale repose sur l'acquisition d'un important jeu de données sur le milieu étudié qu'il s'agira d'interpréter par la suite. Un modèle expérimental permet d'évaluer de façon précise sur un tronçon de rivière donné, la capacité d'autoépuration de manière empirique, par exemple la quantité de tel composé dégradé par unité de longueur du cours d'eau en question. Cependant, ce type de modèle (expérimental) ne permet pas de faire de la prévision à long terme (Cox, 2003), car le fonctionnement d'un cours d'eau peut varier dans le temps. C'est ce que montrent Vagnetti et al. (2003) dans leur étude d'un canal en 2002. Ils ont également montré la difficulté d'interprétation des données pour certains paramètres.

L'approche théorique repose sur une modélisation de l'écosystème. De nombreux modèles existent pour représenter le fonctionnement des écosystèmes: SIMCAT, TOMCAT, QUAL2E, QUASAR, POLMOD.PEST, RIVE, SENEQUE parmi d'autres (Whitehead et al., 1997; Pykh, Malkina-Pykh, 1997 ; Tabuchi et al., 2012). Mais ces modèles ne prennent pas tous en compte les mêmes paramètres pour la mise en équation des procédés de transformation, ni les mêmes procédés et, dans l'ensemble, il n'y a pas de prise en compte des composés chimiques, métaux... Par rapport aux paramètres ceux - ci sont nombreux et peuvent varier dans des fourchettes importantes, ce qui peut entrainer des résultats de simulation relativement éloignés des valeurs réelles (Salla et al, 2016). Cela nécessite, si l'on veut obtenir des résultats plausibles de les caler à partir de mesures réalisées sur le cours d'eau que l'on veut étudier (Salla et al. 2015). De plus, ces modèles exigent de nombreuses informations (notamment en termes de paramètres) sur le système hydrologique qu'ils doivent modéliser, informations qui sont souvent absentes (Manina et al, 2010). Par rapport, aux modèles eux-mêmes, ils s'appuient pour la plupart sur la loi de Fick pour le transport des polluants dans les eaux, bien que des mesures tendent à montrer que les polluants n'obéissent pas totalement à l'advection/dispersion (Benedini, 2011). Les résultats de cette modélisation ne sont pas parfaits.

Finalement, la complexité de l'évaluation et de la modélisation de l'autoépuration met en évidence l'impossibilité actuelle d'évaluer les besoins d'un écosystème aquatique et donc de considérer les écosystèmes aquatiques comme clients. 


\subsection{Identification d'un nouveau client - l'être humain - et utilisation des services écosystémiques}

L'impossibilité d'évaluer les besoins d'un écosystème aquatique de façon simple, rapide et pertinente amène à élargir l'étude du milieu récepteur afin d'identifier un nouveau client apte à exprimer ses besoins : l'être humain est vu comme utilisateur des services rendus par le milieu naturel. Ce sont donc les besoins de l'être humain, en tant que clients secondaires, qui sont pris en considération. Cette nouvelle considération permet de reprendre la figure 2 (pour des raisons de lisibilité, les relations processus fournisseur/ processus client n'ont pas été reproduits). Sur la figure 3 sont représentés les échanges entre les processus écosystémiques (du système «milieu récepteur ») et les processus anthropiques bénéficiaires des services écosystémiques.

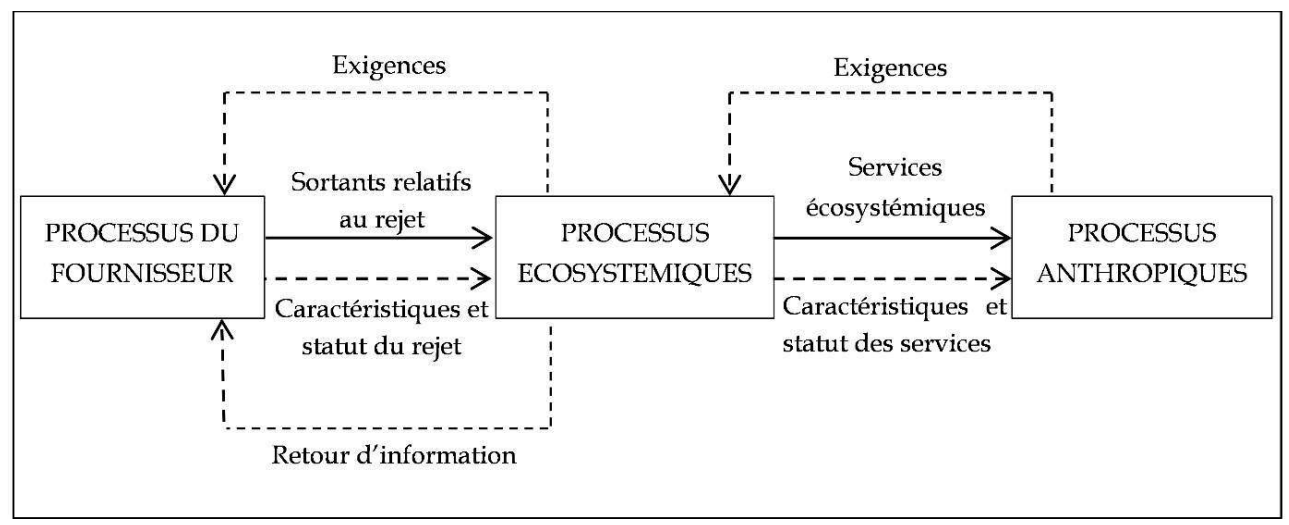

Figure 3 : Schéma conceptuel - élargissement du concept de qualité défini par l'ISO 9000 (2005) aux échanges entre l'environnement et les processus anthropiques bénéficiaires des services écosystémiques produits par les milieux récepteurs

31 Ainsi les exigences des processus anthropiques vis-à-vis des services écosystémiques produits par les processus écosystémiques du milieu récepteur peuvent être évaluées sur la base de paramètres de qualité de l'eau. Sur la base des paramètres choisis pour la réponse à ces besoins, les caractéristiques du milieu récepteur pourront être déterminées pour la satisfaction de ces besoins. Ces caractéristiques du milieu pourront ensuite être utilisées en tant que besoin des écosystèmes constitutifs du milieu récepteur, en particulier pour l'écosystème aquatique récepteur de l'effluent.

Dans ce cadre, les services écosystémiques, qui constituent l'interface entre les écosystèmes et l'humain, permettent une appréhension directe des impacts anthropiques sur les écosystèmes par la modification des services rendus et donc les impacts sur le bien-être de l'humain. En effet, l'humain par son rejet s'impacte lui-même en impactant les écosystèmes sur lesquels il repose en tant que bénéficiaire des services écosystémiques.

Ces réflexions ont permis d'aboutir à une méthodologie d'aide à la gestion des rejets industriels utilisant les services écosystémiques qui sera présentée dans le paragraphe suivant. 


\section{Méthodologie}

\subsection{Objectif et étapes de la méthodologie.}

L'objectif de la méthodologie développée est de dimensionner des rejets aqueux industriels en milieu naturel répondant aux besoins de l'ensemble des parties prenantes dans l'objectif du maintien ou de l'atteinte du bon état des cours d'eau (réponse à la DCE) reposant sur l'identification des services écosystémiques produits sur une zone géographique. Ce dimensionnement des rejets passe par la définition de seuils de rejet et de rendements épuratoires pour l'effluent qui doit être rejeté.

La méthodologie proposée se décompose selon les 4 étapes suivantes :

- Étape 1 : Définition de l'aire d'étude pour le projet

- Étape 2 : Description et analyse de l'aire d'étude

- Étape 3 : Évaluation des impacts du rejet sur l'aire d'étude

- Étape 4 : Évaluation des besoins des cibles, élaboration de seuils de rejet et de rendements épuratoires pour le traitement des eaux usées du projet

Les paragraphes suivants décrivent les différentes étapes de la méthodologie avec un niveau de détail plus important pour les étapes employant les services écosystémiques.

\section{2. Étape 1 : Définition de l'aire d'étude.}

La définition des aires d'étude est une des premières étapes de l'étude d'impact (MEDDE 2010; Michel 2001). Il s'agit de délimiter une ou plusieurs zones, sur la zone géographique entourant le site d'implantation du projet, pour l'étude de ses impacts sur l'environnement.

\section{3. Étape 2 : Description et analyse de l'aire d'étude.}

Dans cette étape, l'aire d'étude définie à l'étape précédente est analysée du point de vue de sa composition (étape 2.1) et des services écosystémiques produits par ses composantes (étape 2.2).

\subsection{1. Étape 2.1 Identification de l'ensemble des composantes de l'aire d'étude}

Il s'agit d'identifier, de différencier et de décrire les différentes parties ou écosystèmes qui composent l'aire d'étude. L'outil de base utilisé pour cette analyse est la représentation paysagère de l'aire d'étude sous Corine Land Cover (CLC). Pour la représentation des cours d'eau, cette cartographie peut être complétée par exemple par l'emploi des Systèmes d'Information sur l'Eau (SIE: http://www.eaufrance.fr/ comprendre/les-donnees-sur-l-eau/?id_article=833) des différents bassins hydrographiques. À l'issu de cette étape, deux éléments sont obtenus :

- une carte de la zone géographique et de ses composantes hydrographiques et paysagères,

- une liste des composantes de l'aire identifiées. 


\subsection{2. Étape 2.2 Identification des services produits par les composantes de l'aire d'étude : les services écosystémiques}

Plusieurs définitions et catégorisations des services écosystémiques se retrouvent dans la littérature. Ainsi, les services écosystémiques peuvent se catégoriser comme intermédiaires (fonctions, processus écologiques, entités biophysiques associées) ou finaux (composante de la biodiversité directement utilisée, consommée, appréciée par les humais) (Fisher et al, 2009). Cependant, ces catégorisations peuvent s'avérer différentes en fonction des auteurs (Couvet, 2016). L'idée ici n'est pas de donner une nouvelle définition des services, mais de s'assurer de la faisabilité de prendre en considération la notion de service écosystémique dans les processus de prise de décision. Ainsi, nous avons choisi de retenir la définition des «services écosystémiques majeurs » du MEA bien que les services proposés soient de dimensions hétérogènes.

Le MEA inventorie et catégorise les différents services rendus par les écosystèmes. Ceuxci se divisent en quatre groupes (MEA, 2005):

- Les services support «nécessaires à tous les autres services écosystémiques » : formation du sol, cycle des nutriments, production primaire. Ces services sont également appelés « intégrité de l'écosystème » par certaines communautés (Burkhard et al., 2009);

- Les services de régulation qui sont les «bénéfices engendrés par la régulation des processus écosystémiques » : la régulation du climat, des maladies, de l'eau, la purification de l'eau, la pollinisation ;

- Les services d'approvisionnement de "produits fournis par les écosystèmes » : nourriture, eau pure, bois de chauffage, fibres, biochimie, ressources génétiques ;

- Les services culturels constituent les «bénéfices immatériels apportés par les écosystèmes » : spirituels et religieux, les loisirs et l'écotourisme, l'esthétique, l'inspiration, l'éducation, sentiment d'appartenance, héritage culturel.

Cependant, suivant la source bibliographique, certains services ne se trouvent pas toujours dans la même catégorie, par exemple, les cycles nutritifs sont cités comme services de support par le Millenium Ecosystem Assessment, par Burkhard et al (2009), ainsi que par Baker et al (2012), mais sont classés comme service de régulation dans le rapport de l'ONEMA (Wallis et al., 2011). Les différents services écosystémiques peuvent également être différents dans le détail suivant les auteurs.

Pour la suite de ce travail, seuls les services écosystémiques répondant de manière directe à un besoin de l'humain seront conservés. Certains services "supports ", qui relèvent du fonctionnement intrinsèque des écosystèmes, difficilement appréhendables de manière quantitative en termes de besoin, ont été écartés de l'étude. Ainsi, nous avons choisi de ne pas traiter les services tels que la pollinisation, les cycles nutritifs, la capture d'exergie. De plus, certaines dénominations ont été adaptées. (Grazilhon, 2015)

Afin d'apporter une représentation structurée des services écosystémiques et d'en assurer une utilisation dans le cadre de la prise de décision au regard des composantes de l'aire d'étude, les services fournis par les différents types d'écosystèmes sont représentés dans une matrice des services, à la manière de Burkhard (Burkhard et al., 2009). Cette matrice (tableau 2) permet de :

- sélectionner uniquement les composantes présentes sur l'aire d'étude (correspondant aux unités paysagères CLC identifiées à l'étape 2.1), 
- lister et nommer de manière exhaustive les différentes composantes de la zone géographique.

Tableau 2 : Matrice des services écosystémiques produits par les composantes du milieu récepteur

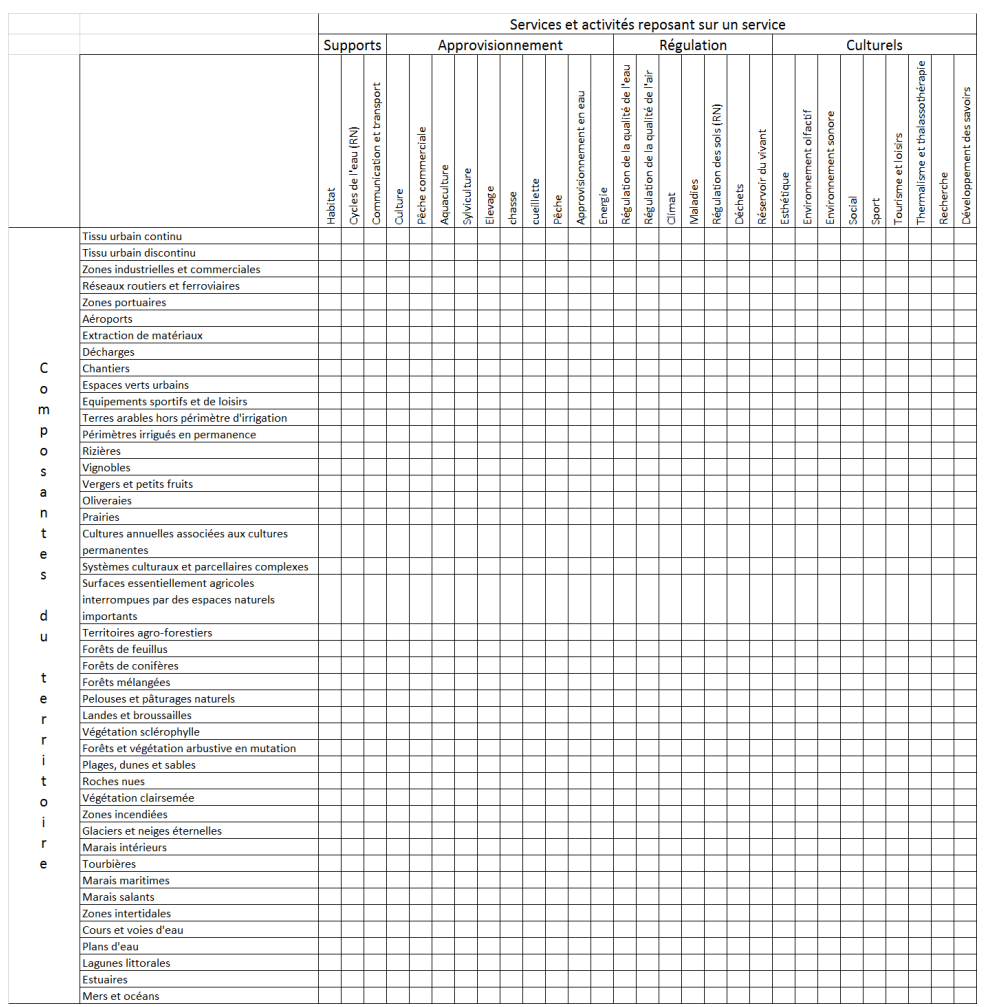

En outre, les services produits et les services non produits pour chacune des composantes de l'aire d'étude à l'étape 2.1 doivent être identifiés. Les éléments permettant d'attribuer un service réellement rendu par une composante territoriale relèvent d'une étude de terrain. À l'issue de cette étude locale, les services produits par les différentes composantes sont représentés dans la matrice par une notation à échelle binaire inscrite à chaque intersection service/composante :

- 0 = le service n'est pas produit par cette composante ;

- 1 = le service est produit par cette composante.

Une matrice "portrait » des composantes de l'aire d'étude et des services qu'elles fournissent est ainsi obtenue pour la zone géographique mise en jeu.

\section{4. Étape 3 : Évaluation des dommages liés aux impacts du projet sur l'aire d'étude.}

L'évaluation des dommages causés par le rejet sur la zone géographique nécessite en premier lieu l'étude des flux d'eau, vecteurs de l'impact (étape 3.1), puis l'étude de l'évolution sur le réseau hydrographique de l'aire d'étude des composés véhiculés par ce vecteur et l'identification des composantes susceptibles de subir des effets (étape 3.2). Enfin, les différents impacts ainsi que les cibles et parties prenantes associées sont identifiées (étape 3.3). 


\subsection{1. Étape 3.1 Analyse des flux d'eau sur l'aire d'étude.}

49 d'étude afin d'identifier les composantes/écosystèmes pouvant subir des effets suite au rejet. Une fois ces composantes identifiées, cette étape va consister à évaluer la possibilité d'effets ou non sur les différents services fournis par ces composantes.

expliqué dans le paragraphe 1 , les phénomènes d'autoépuration ne sont pas considérés. Les modifications qu'implique un rejet sur les masses d'eaux réceptrices et avals sont évaluées par calculs de dilution simple qui ne sont pas détaillés dans cet article.

\subsection{3. Étape 3.3 Identification des dommages potentiels, des cibles et des parties prenantes.}

\section{Identification des services potentiellement dommageables des différentes composantes}

Dans un premier temps, la démarche consiste à identifier les services fournis par les différentes composantes qui peuvent être affectés par les impacts de la modification du milieu. Ces services peuvent subir des dommages de manière directe ou indirecte (causés par un ou plusieurs dommages directs). En matérialisant ces dommages par des flèches, un arbre des conséquences permettant de visualiser le spectre des dommages liés à la modification physicochimique du milieu récepteur peut être construit sur la matrice eau. La construction de l'arbre des dommages démarre à partir de l'élément déclencheur : dans l'étude des impacts des rejets d'eaux usées, l'élément déclencheur est la modification du milieu dans lequel l'effluent est rejeté. Cette modification se traduit en termes quantitatifs et qualitatifs (effets évalués à l'étape 3.2). Elle entraîne potentiellement en premier lieu des dommages sur les services fournis par le milieu récepteur et en second lieu sur les services fournis par les autres composantes de la matrice « portrait-eau ».

\section{Identification des cibles et des parties prenantes}

Dans un deuxième temps, pour chaque service de chaque composante pouvant subir des dommages, il est alors possible d'identifier les cibles: ce sont les bénéficiaires de ces services. L'identification des cibles se fait sur le terrain, et/ou en concertation avec des 
experts (bureaux d'étude, DREAL, etc.). Après l'identification des cibles associées aux services écosystémiques impactés, il est important d'identifier les parties prenantes susceptibles de les représenter dans les processus décisionnels.

De cette troisième étape, un arbre des dommages/conséquences du rejet sur son aire d'étude et pour chaque composante concernée est proposé; il reprend les services pouvant subir des dommages, les cibles et leurs parties prenantes.

\section{5. Étape 4 : Évaluation des besoins des cibles, élaboration de seuils de rejets et de rendements épuratoire pour le traitement des eaux usées du projet}

\subsection{1. Étape 4.1 Évaluation des besoins des cibles vis-à-vis des milieux récepteurs}

L'évaluation des besoins des cibles se fait au regard des services potentiellement dommageables et des cibles identifiées. Le tableau 3 présente une liste non exhaustive des services potentiellement dommageables suite à un rejet d'eaux usées dans un cours d'eau. L'évaluation de la capacité du milieu à répondre aux différents besoins peut se faire à l'aide de plusieurs normes ou textes réglementaires qui sont ou qui ont été en vigueur en France :

- le bon état des masses d'eau défini par la Directive Cadre sur l'Eau (DCE) et dont les normes sont fixées en France par l'arrêté du 25/01/2010,

- les Normes de Qualité Environnementale (NQE) pour les substances dangereuses et les substances dangereuses prioritaires, ainsi que les NQE proposées par l'INERIS pour un certain nombre d'autres composés,

- la SEQ eau (SEQ), qui n'est plus en vigueur, mais qui propose pour différentes catégories de vocation du milieu (abreuvage, production d'eau potable, irrigation, loisirs, aquaculture) des normes à respecter pour différents paramètres.

Tableau 3 : Exemples de services pouvant être impactés par des rejets d'eaux usées et besoins spécifiques relatifs à ces services

\begin{tabular}{|l|l|l|}
\hline $\begin{array}{l}\text { Service dommageable } \\
\text { par rejet d'effluent dans } \\
\text { une masse d'eau }\end{array}$ & $\begin{array}{l}\text { Besoins des cibles vis-à-vis de ces } \\
\text { services }\end{array}$ & $\begin{array}{l}\text { Critères d'évitement } \\
\text { des dommages } \\
\text { existants }\end{array}$ \\
\hline Habitat & $\begin{array}{l}\text { Nécessité de maintenir un habitat de } \\
\text { bonne qualité pour les espèces } \\
\text { endémiques en état (DCE) }\end{array}$ & Bon \\
\hline Pêche commerciale & $\begin{array}{l}\text { Qualité de l'eau garantissant l'habitat et la } \\
\text { santé de la faune aquatique, ainsi que la } \\
\text { non-contamination des consommateurs }\end{array}$ & $\begin{array}{l}\text { Bon état (DCE), SEQ } \\
\text { aquaculture }\end{array}$ \\
\hline Aquaculture & $\begin{array}{l}\text { Qualité de l'eau garantissant l'habitat et la } \\
\text { santé de la faune aquatique, ainsi que la } \\
\text { non-contamination des consommateurs }\end{array}$ & SEQ aquaculture, NQE \\
\hline
\end{tabular}




\begin{tabular}{|c|c|c|}
\hline Pêche & $\begin{array}{l}\text { Qualité de l'eau garantissant l'habitat et la } \\
\text { santé de la faune aquatique, ainsi que la } \\
\text { non-contamination des consommateurs }\end{array}$ & SEQ aquaculture, $\mathrm{NQE}$ \\
\hline $\begin{array}{l}\text { Régulation de la qualité de } \\
\text { l'eau }\end{array}$ & $\begin{array}{l}\text { De l'eau en qualité et en quantité } \\
\text { nécessaires pour assurer les phénomènes } \\
\text { d'autoépuration }\end{array}$ & $\begin{array}{l}\text { Bon état écologique } \\
\text { (DCE), } \mathrm{NQE}\end{array}$ \\
\hline Approvisionnement en eau & $\begin{array}{l}\text { Qualité de l'eau pour les différents types } \\
\text { d'approvisionnement: eau potable, eau } \\
\text { d'irrigation, eau d'abreuvage, eau } \\
\text { industrielle, eau pour l'aquaculture }\end{array}$ & $\begin{array}{l}\text { Bon état (DCE), SEQ } \\
\text { abreuvage, production } \\
\text { d'eaurable, } \\
\text { irrigation, aquaculture }\end{array}$ \\
\hline Régulation du climat & $\begin{array}{l}\text { Lié à « Régulation de la qualité de l'eau ", } \\
\text { en particulier sur la captation et le } \\
\text { relargage de gaz à effet de serre }\end{array}$ & Bon état (DCE) \\
\hline Maladies & $\begin{array}{l}\text { Nécessité de ne pas répandre des } \\
\text { pathogènes, ne pas créer un milieu } \\
\text { propice à leur développement }\end{array}$ & Bon état (DCE) \\
\hline Cycles de l'eau & $\begin{array}{l}\text { Quantité d'eau rejetée, non colmatage de } \\
\text { l'écosystème }\end{array}$ & - \\
\hline Déchets & $\begin{array}{l}\text { Voir «Qualité de l'eau », la qualité du } \\
\text { milieu permet l'assimilation et l'épuration } \\
\text { des effluents qu'il reçoit }\end{array}$ & - \\
\hline Réservoir du vivant & $\begin{array}{l}\text { Respect de la biodiversité, d'espèces } \\
\text { locales particulières, rares ou protégées }\end{array}$ & Bon état (DCE), NQE \\
\hline Esthétique & \multirow{2}{*}{ Liés à la régulation de la qualité de l'eau } & Bon état (DCE) \\
\hline Environnement olfactif & & Bon état (DCE) \\
\hline Social & $\begin{array}{l}\text { Suivant enjeux locaux, lié au bon } \\
\text { fonctionnement de l'écosystème }\end{array}$ & Bon état (DCE) \\
\hline Sport & Qualité de l'eau pour la baignade & Bon état, SEQ loisirs \\
\hline Tourisme et loisirs & $\begin{array}{l}\text { Qualité de l'eau pour la baignade, aspects } \\
\text { esthétiques, olfactifs, pêche de loisirs... }\end{array}$ & $\begin{array}{l}\text { Bon état (DCE), SEQ } \\
\text { loisirs }\end{array}$ \\
\hline $\begin{array}{l}\text { Thermalisme et } \\
\text { thalassothérapie }\end{array}$ & Non contamination des eaux souterraines & - \\
\hline
\end{tabular}

57 Les valeurs les plus discriminantes pour chaque paramètre sont choisies comme concentrations maximales à ne pas dépasser dans chaque milieu pouvant potentiellement subir des effets. 


\subsection{2. Étape 4.2 Détermination des seuils de rejet acceptables et de rendement épuratoire pour le traitement des eaux usées du projet} Frères situé près de Bordeaux, afin d'évaluer les impacts d'un rejet des effluents aqueux dans le milieu naturel. Du fait de son activité et du volume de production, l'usine est une Installation Classée pour la Protection de l'Environnement (ICPE) d'après plusieurs rubriques.

61 Les différentes étapes de la méthodologie sont développées dans les paragraphes suivants.

\section{1. Étape 1 Définition de l'aire d'étude}

63 La carte (figure 4) montre le réseau hydrographique de la zone d'étude avec un axe principal, le fleuve de la Garonne et un réseau secondaire dont fait partie le cours d'eau dans lequel le rejet est prévu pour le projet : la Jalle de Blanquefort, au sud du site.

\section{2 Étape 2 description et analyse de l'aire d'étude}

\subsection{1 Étape 2.1 : Identification de l'ensemble des composantes de l'aire d'étude}

64 L'aire d'étude est analysée selon une étude cartographique. Visuellement, les photographies aériennes montrent un paysage essentiellement agricole avec quelques zones urbaines et industrielles au niveau de Blanquefort et de la rive droite du fleuve. Plusieurs plans d'eau se trouvent proches du site. Le réseau hydrographique de surface apparait avec le fleuve, La Garonne, et notamment la Jalle de Blanquefort qui est destinée à recevoir les effluents du site (figure 4).

65 Afin de connaitre plus précisément les écosystèmes et de manière générale les composantes de l'aire d'étude, la zone géographique est visualisée sur une carte représentant les unités paysagères de Corine Land Cover (cartographie de 2006) et l'hydrologie de surface comme le montre la figure 4. 


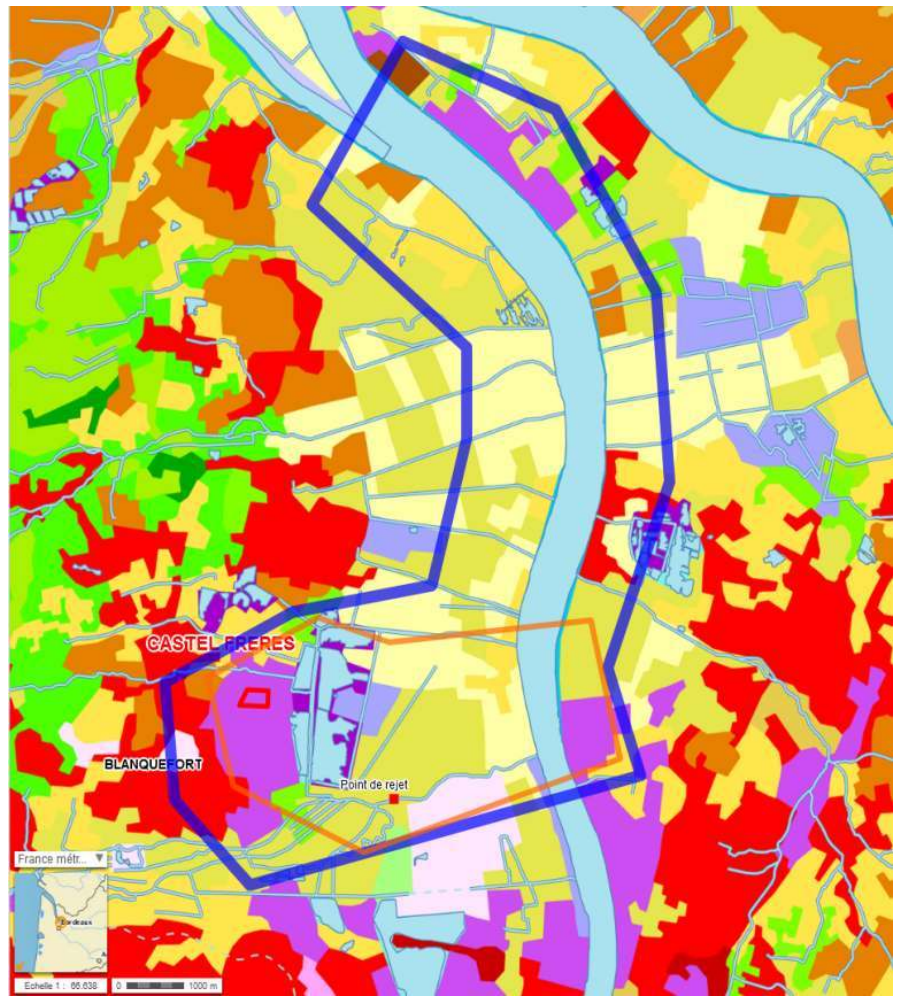

Figure 4 : Couverture Corine Land Cover 2006 et hydrologie de surface sur l'aire d'étude (Légende tableau 4) (IGN 2015)

Tableau 4 (série): Nomenclature et légende de la représentation cartographique Corine Land Cover (Bossard, Feranec, Otahel 2000)

\begin{tabular}{|l|}
\hline \multicolumn{1}{|l|}{ Unités paysagères de Corine Land Cover } \\
\hline 1 Territoires artificialisés \\
\hline 11 Zones urbanisées \\
\hline 111 Tissu urbain continu \\
\hline 112 Tissu urbain discontinu \\
\hline 12 Zones industrielles ou commerciales et réseaux de \\
communication \\
\hline 121 Zones industrielles et commerciales \\
\hline 122 Réseaux routier et ferroviaire et espaces associés \\
\hline 123 Zones portuaires \\
\hline 124 Aéroports \\
\hline 13 Mines, décharges et chantiers \\
\hline 131 Extraction de matériaux \\
\hline 132 Décharges \\
\hline 133 Chantiers \\
\hline 14 Espaces verts artificialisés, non agricoles \\
\hline 141 Espaces verts urbains \\
\hline 142 Équipements sportifs et de loisirs \\
\hline
\end{tabular}




\begin{tabular}{|c|}
\hline Unités paysagères de Corine Land Cover \\
\hline 2 Territoires agricoles \\
\hline 21 Terres arables \\
\hline 211 Terres arables hors périmètres d'irrigation \\
\hline 212 Périmètres irrigués en permanence \\
\hline 213 Rizières \\
\hline 22 Cultures permanentes \\
\hline 221 Vignobles \\
\hline 222 Vergers et petits fruits \\
\hline 223 Oliveraies \\
\hline 23 Prairies \\
\hline 231 Prairies \\
\hline 24 Zones agricoles hétérogènes \\
\hline $\begin{array}{l}241 \text { Cultures annuelles associées aux cultures } \\
\text { permanentes }\end{array}$ \\
\hline 242 Systèmes culturaux et parcellaires complexes \\
\hline $\begin{array}{l}243 \text { Surfaces essentiellement agricoles, interrompues } \\
\text { par des espaces naturels importants }\end{array}$ \\
\hline 244 Territoires agroforestiers \\
\hline
\end{tabular}

\begin{tabular}{|l|l|}
\hline \multicolumn{1}{|l|}{ Unités paysagères de Corine Land Cover } \\
\hline \multicolumn{1}{|l}{ Forêts et milieux semi-naturels } \\
\hline 31 Forêts \\
\hline 311 Forêts de feuillus \\
\hline 312 Forêts de conifères \\
\hline 313 Forêts mélangées \\
\hline 32 Milieux à végétation arbustive et/ou herbacée \\
\hline 321 Pelouses et pâturages naturels \\
\hline 322 Landes et broussailles \\
\hline 323 Végétation sclérophylle \\
\hline 324 Forêt et végétation arbustive en mutation \\
\hline 33 Espaces ouverts, sans ou avec peu de végétation \\
\hline 331 Plages, dunes et sable \\
\hline 332 Roches nues \\
\hline 333 Végétation clairsemée \\
\hline 334 Zones incendiées \\
\hline 335 Glaciers et neiges éternelles \\
\hline
\end{tabular}




\section{Unités paysagères de Corine Land Cover}

\section{Zones humides}

\section{Zones humides intérieures}

411 Marais intérieurs

412 Tourbières

42 Zones humides maritimes

421 Marais maritimes

422 Marais salants

423 Zones intertidales

Unités paysagères de Corine Land Cover

5 Surfaces en eau

51 Eaux continentales

511 Cours et voies d'eau

512 Plans d'eau

\section{Eaux maritimes}

521 Lagunes littorales

522 Estuaires

523 Mers et océans

4.2.2. Étape 2.2 : Identification des services produits par les composantes de l'aire d'étude

En partant de la matrice générique, seules les composantes de l'aire d'étude sont conservées dans la colonne gauche de la matrice des services (identifiées à l'étape 2.1). Ainsi la matrice-portait est réalisée en associant la note de 1 si le service est rendu pour la composante et la note de 0 si le service n'est pas rendu. Le tableau 5 présente la matriceportrait des services écosystémiques potentiels apportés pour le cas d'étude. 
Tableau 5 : Matrice portrait des services écosystémiques potentiels sur l'aire d'étude

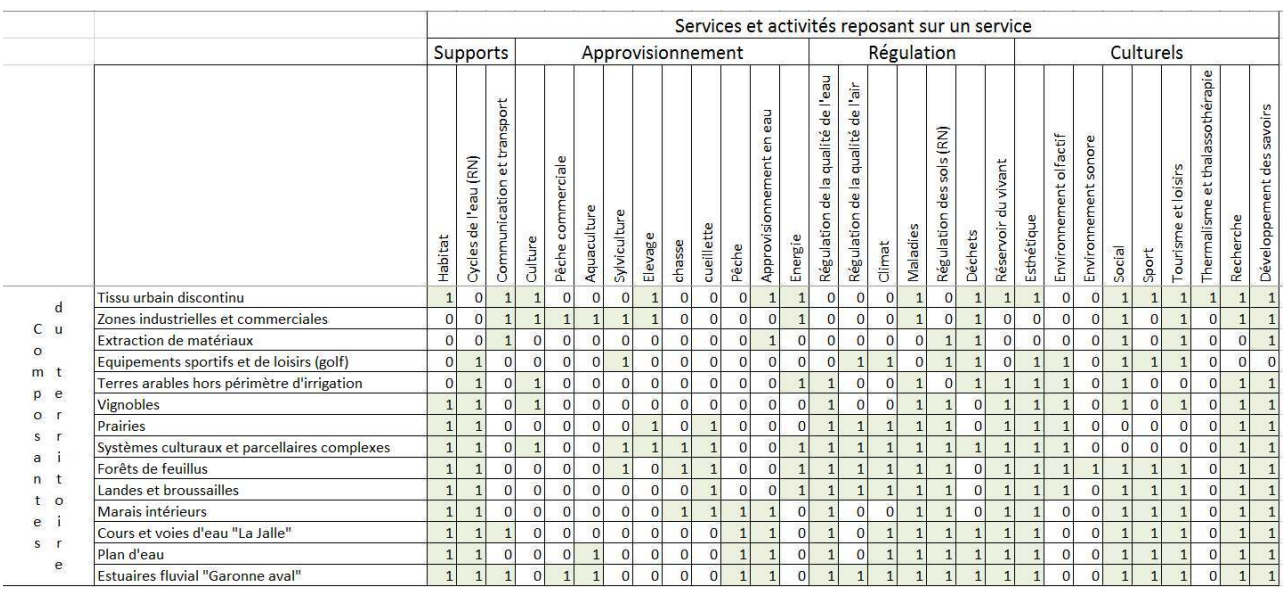

\section{3. Étape 3 Évaluation des dommages potentiels liés aux impacts du projet sur l'aire d'étude}

\subsection{1. Étape 3.1 Analyse des flux d'eau sur l'aire d'étude}

67 L'analyse des flux d'eau s'effectue via la représentation des liens entre les masses d'eau superficielles et souterraines avec les systèmes anthropiques et écosystème de la zone d'étude. La réalisation du diagramme de flux d'eau de surface repose sur des données de prélèvements et de rejets provenant du site internet du SIE Adour Garonne (figure 5).

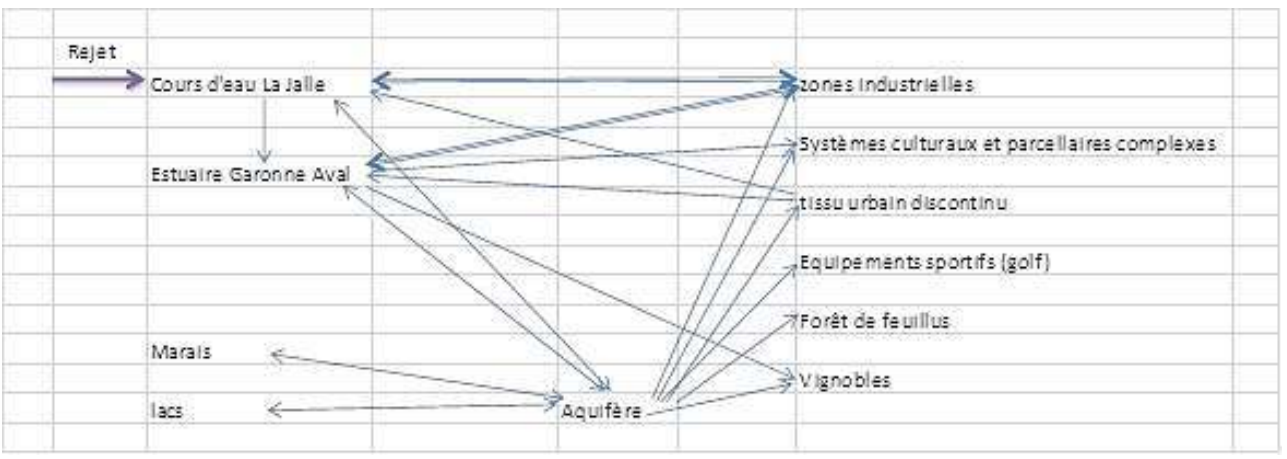

Figure 5 : Diagramme des flux d'eau sur l'aire d'étude

Les composantes retenues au final sont alors sélectionnées (zones industrielles, systèmes culturaux et parcellaires complexes, vignobles) et les flux vers l'aval du projet sont représentés dans la matrice " portrait-eau », sur la figure 6 . 


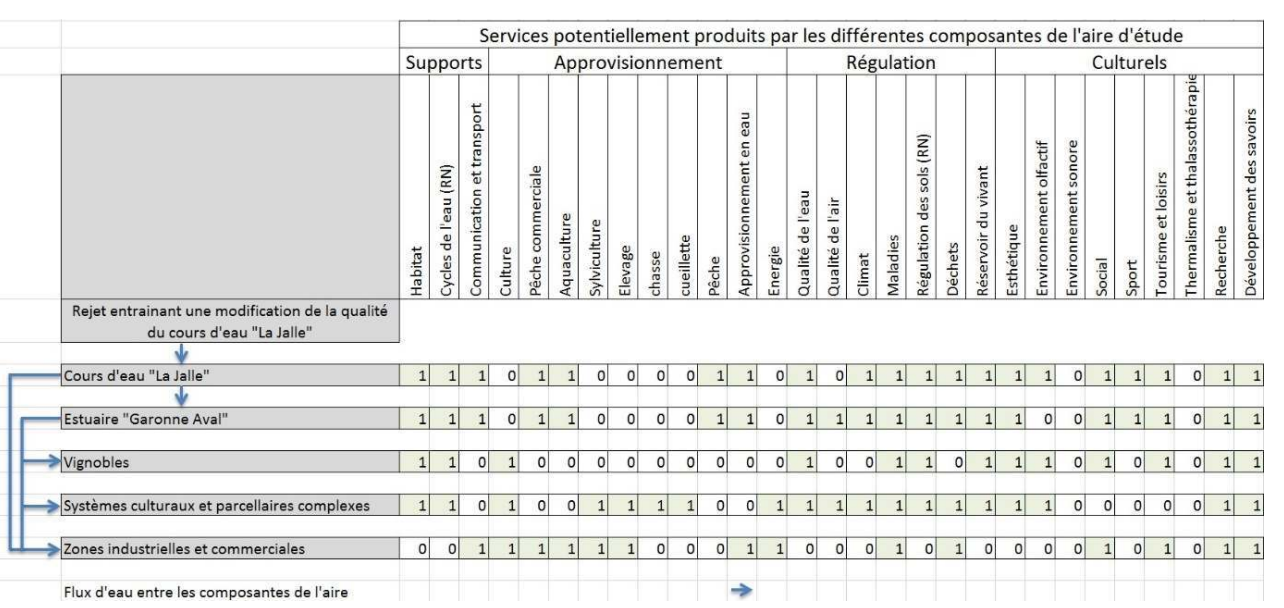

Figure 6 : Matrice « portrait - eau » de l'aire d'étude

\subsection{2. Étape 3.2 Étude des effets induits par le rejet}

Dans le cadre de la règlementation ICPE et IED, l'entreprise Castel Frères est soumise pour son site de Blanquefort à la surveillance de ses effluents. Les effluents d'origine viticole émis par l'entreprise sont essentiellement organiques. Les effets induits par le rejet sur les masses d'eau aval pour chaque paramètre physico-chimique classique (DCO, $\mathrm{DBO}_{5}$, azote, phosphore) sont étudiés. Les effets sont obtenus par calcul de dilution sur les masses d'eau en aval (tableau 6).

Tableau 6 : Mesure des effets sur les concentrations de différents paramètres dans les masses d'eau en aval du rejet (non traité) par calculs de flux (en rouge : les paramètres pour lesquels il a été possible de calculer les effets) ( $"-»=$ donnée non disponible)

\begin{tabular}{|c|c|c|c|c|c|c|}
\hline Paramètre & $\begin{array}{l}\text { La Jalle de } \\
\text { Blanquefort } \\
\text { (concentrations } \\
\text { moyenne } \\
\text { annuelle 2012) } \\
\text { (SIE A-G) }\end{array}$ & $\begin{array}{l}\text { Effluents Castel } \\
-\quad \text { Blanquefort } \\
\text { (concentrations } \\
\text { moyennes } \\
\text { annuelles 2011) }\end{array}$ & $\begin{array}{l}\text { Calcul des } \\
\text { effets sur la } \\
\text { Jalle : } \\
\text { Jalle de } \\
\text { Blanquefort } \\
+\quad \text { effluent } \\
\text { Castel }\end{array}$ & $\begin{array}{l}\text { L'estuaire } \\
\text { fluvial Garonne } \\
\text { aval (aval de la } \\
\text { confluence } \\
\text { avec la Jalle) } \\
\text { (concentrations } \\
\text { moyennes } \\
\text { annuelles 2012) } \\
\text { (SIE A-G) }\end{array}$ & $\begin{array}{l}\text { Calcul } \\
\text { des effets } \\
\text { sur la } \\
\text { Garonne : } \\
\text { Estuaire } \\
\text { fluvial } \\
\text { Garonne } \\
\text { aval+ } \\
\text { effluent } \\
\text { Castel }\end{array}$ & $\begin{array}{l}\text { Seuil du } \\
\text { bon état } \\
\text { (arrêté du } \\
25 / 01 / 2010)\end{array}$ \\
\hline Débit (m3/j) & 5270 & 273 & $\begin{array}{l}5270+273= \\
5543\end{array}=$ & $5,62.10^{7}$ & $5,62.10^{7}$ & \\
\hline DCO $(\mathrm{mg} / \mathrm{L})$ & - & 3683 & - & - & - & $<7$ \\
\hline $\mathrm{DBO}_{5}(\mathrm{mg} / \mathrm{L})$ & 1.6 & 1935 & 97 & 1.5 & 1.5 & $<6$ \\
\hline $\begin{array}{l}\text { Oxygène Dissous } \\
(\mathrm{mg} / \mathrm{L})\end{array}$ & 6.9 & - & - & 8.6 & - & $>6$ \\
\hline
\end{tabular}




\begin{tabular}{|c|c|c|c|c|c|c|}
\hline $\begin{array}{ll}\text { Taux } & \text { de } \\
\text { saturation } & \text { en } \\
\text { oxygène \% } & \end{array}$ & 89 & - & - & 83 & - & $>70$ \\
\hline $\begin{array}{l}\text { Ammonium }\left(\mathrm{NH}_{4}\right. \\
+)(\mathrm{mg} / \mathrm{L})\end{array}$ & 0.3 & - & - & 0.4 & - & $<0.5$ \\
\hline $\begin{array}{ll}\text { Nitrites } & \left(\mathrm{NO}_{2}^{-}\right) \\
(\mathrm{mg} / \mathrm{L}) & \end{array}$ & 0.11 & - & - & 0.07 & - & $<0.3$ \\
\hline $\begin{array}{ll}\text { Nitrates } & \left(\mathrm{NO}_{3}^{-}\right) \\
(\mathrm{mg} / \mathrm{L}) & \end{array}$ & 7.5 & - & - & 8 & - & $<50$ \\
\hline $\begin{array}{l}\text { Azote Kjeldhal } \\
(\mathrm{NKJ})(\mathrm{mg} / \mathrm{L})\end{array}$ & - & 20 & - & - & - & - \\
\hline $\begin{array}{l}\text { Phosphore total } \\
\text { (PT) }(\mathrm{mg} / \mathrm{L})\end{array}$ & 0.11 & 11 & 0.64 & 0.57 & 0.57 & $<0.2$ \\
\hline $\begin{array}{l}\text { Orthophosphates } \\
\left(\mathrm{PO}_{4}{ }^{3-}\right)(\mathrm{mg} / \mathrm{L})\end{array}$ & 0.13 & - & - & 0.18 & - & $<0.5$ \\
\hline pH min & 7.1 & - & - & 7.6 & - & \\
\hline pH max & 8 & - & - & 8.31 & - & \\
\hline
\end{tabular}

\subsection{3. Étape 3.3 Identification des dommages potentiels, des cibles et des parties prenantes}

\section{Identification des services potentiellement dommageables sur les différentes composantes}

L'effluent étant principalement organique et biodégradable, l'augmentation de la concentration de ces composés dans le milieu va avoir pour effet des problèmes de mauvaise oxygénation dans le milieu, du fait principalement que La Jalle est un cours d'eau à faible débit et à étiage prononcé. Un tel phénomène peut causer des pressions sur l'écosystème et des dysfonctionnements, en particulier l'asphyxie de la faune aquatique (CNRS 2014). Ce phénomène entraine donc des impacts directs sur la pêche du fait, soit de l'extinction de certains individus, soit de leur migration dans des lieux plus propices (perte d'habitat). La prolifération d'algues peut également survenir suite à un excès de nutriments dans le milieu. Ceci entraine généralement un arrêt du fonctionnement de l'écosystème plus ou moins réversible avec pour conséquences pour l'être humain, d'une part, une dégradation de la qualité esthétique et olfactive du cours d'eau. D'autre part, cela pourra entrainer des problématiques pour les composantes de la zone géographique qui s'approvisionnent en eau dans La Jalle. Dans le cas présent, il s'agit des zones urbaines et industrielles.

La Jalle étant un affluent de la Garonne, la modification de sa qualité va potentiellement entrainer, comme évoqué précédemment, des effets mesurables sur la qualité de l'eau de 
la Garonne avec possiblement une hausse de la demande en oxygène. Cependant, la Garonne a un très fort débit à la confluence de La Jalle, l'augmentation de la demande en oxygène sera relativement faible et n'aura donc pas les mêmes conséquences sur l'écosystème que sur celui de la Jalle. Ces constatations permettent d'identifier les services impactés (figure 7). La figure 7 présente l'arborescence des dommages occasionnés par le rejet de l'entreprise sur les différentes composantes de la zone géographique et des services. Les flèches rouges matérialisent cette arborescence.

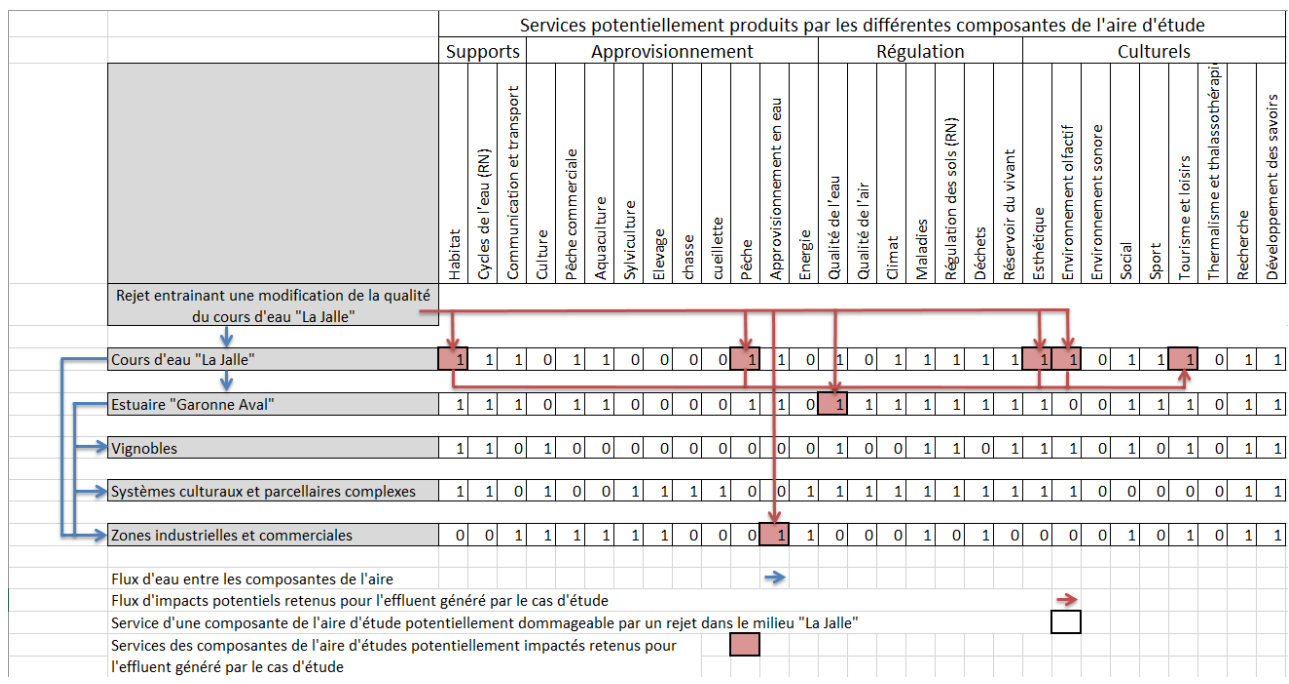

Figure 7 : Arbre de dommage : cheminement des impacts sur les services écosystémiques au sein de l'aire d'étude

\section{Identification des cibles et des parties prenantes}

L'identification des cibles et des parties prenantes permet de mettre en exergue les acteurs impactés ou impliqués par les changements potentiels qu'engendrerait le rejet de l'entreprise.

De par son activité et ses rejets aqueux, l'entreprise est déjà en relation directe avec certains acteurs sur sa zone géographique :

- Le préfet de département qui a prononcé l'autorisation d'exploiter de l'entreprise,

- La Communauté Urbaine de Bordeaux (CUB), qui est propriétaire de la station d'épuration des eaux usées de la commune de Blanquefort et qui détermine actuellement les niveaux autorisés pour le rejet dans la station, ainsi que les opérateurs de la station : la Lyonnaise des eaux,

- La DREAL qui contrôle la conformité de l'installation à son arrêté d'autorisation,

- L'Agence de l'eau Adour Garonne dans le cadre de la déclaration des émissions, du paiement de la redevance et des demandes de subventions.

- L'association de protection de la nature qui est en charge du site Natura 2000 « Marais de Bruges ».

Les cibles sont identifiées pour chaque service affecté en fonction de la composante concernée. Il est ainsi possible d'établir une corrélation entre les composantes impactées, les services concernés, les cibles affectées et les parties prenantes impliquées dans le processus de décision (tableau 7). 
Tableau 7 : Parties prenantes impliquées dans les impacts du rejet sur sa zone géographique

\begin{tabular}{|c|c|c|c|}
\hline $\begin{array}{l}\text { Composantes impactées par } \\
\text { la modification de la } \\
\text { qualité de La Jalle }\end{array}$ & Services concernés & Cibles Identifiées & $\begin{array}{l}\text { Parties prenantes } \\
\text { associées }\end{array}$ \\
\hline \multirow{5}{*}{ La Jalle de Blanquefort } & Habitat & \multirow{2}{*}{$\begin{array}{l}\text { Pêcheurs, } \\
\text { associations de } \\
\text { pêche }\end{array}$} & \multirow{2}{*}{$\begin{array}{l}\text { Associations } \quad \mathrm{de} \\
\text { pêche }\end{array}$} \\
\hline & Pêche & & \\
\hline & Esthétique & \multirow{3}{*}{$\begin{array}{l}\text { Habitants, } \\
\text { promeneurs, } \\
\text { touristes }\end{array}$} & \multirow{3}{*}{$\begin{array}{l}\text { Communauté } \\
\text { communes }\end{array}$} \\
\hline & $\begin{array}{l}\text { Environnement } \\
\text { olfactif }\end{array}$ & & \\
\hline & Tourisme et loisirs & & \\
\hline $\begin{array}{l}\text { Les zones industrielles et } \\
\text { commerciales }\end{array}$ & $\begin{array}{l}\text { Approvisionnement } \\
\text { en eau }\end{array}$ & $\begin{array}{l}\text { Industriels } \\
\text { concernés }\end{array}$ & $\begin{array}{l}\text { Syndicats mixtes, } \\
\text { associations } \\
\text { d'industriels }\end{array}$ \\
\hline $\begin{array}{l}\text { L'estuaire fluvial Garonne } \\
\text { aval }\end{array}$ & Qualité de l'eau & - & $\begin{array}{l}\text { Agence de l'eau, } \\
\text { DREAL }\end{array}$ \\
\hline
\end{tabular}

\section{4. Étape 4 Évaluation des besoins des cibles, élaboration de seuils de rejets et de rendements épuratoires pour le traitement des eaux usées du projet}

\subsection{1. Étape 4.1 : Évaluation des besoins des cibles vis-à-vis du milieu récepteur}

Pour chaque cible impactée par une modification des services, les besoins sont identifiés et répertoriés dans le tableau 8 . La relation entre ces éléments et les critères d'évitement issus des normes et textes règlementaires permettent de définir les seuils à respecter pour assurer un bon état des masses d'eau

Tableau 8 : Services potentiellement dommageables sur la zone d'étude et critères d'évitements des dommages

\begin{tabular}{|l|l|l|l|}
\hline $\begin{array}{l}\text { Composantes } \\
\text { impactées par la } \\
\text { modification de la } \\
\text { qualité de la Jalle } \\
\text { de Blanquefort }\end{array}$ & $\begin{array}{l}\text { Services } \\
\text { dommageables par } \\
\text { rejet d'effluent dans la } \\
\text { Jalle de Blanquefort }\end{array}$ & $\begin{array}{l}\text { Besoins des cibles vis-à-vis } \\
\text { de ces services }\end{array}$ & $\begin{array}{l}\text { Critères } \\
\text { d'évitement } \\
\text { des dommages } \\
\text { existants }\end{array}$ \\
\hline $\begin{array}{l}\text { La Jalle de } \\
\text { Blanquefort }\end{array}$ & Habitat & $\begin{array}{l}\text { Nécessité de maintenir un } \\
\text { habitat de bonne qualité pour } \\
\text { les espèces endémiques }\end{array}$ & Bon état (DCE) \\
\hline
\end{tabular}




\begin{tabular}{|c|c|c|c|}
\hline & Pêche & $\begin{array}{l}\text { Qualité de l'eau garantissant } \\
\text { l'habitat et la santé de la } \\
\text { faune aquatique, ainsi que la } \\
\text { non-contamination des } \\
\text { consommateurs }\end{array}$ & $\begin{array}{l}\text { SEQ } \\
\text { aquaculture, } \\
\text { NQE }\end{array}$ \\
\hline & Esthétique & \multirow{2}{*}{$\begin{array}{l}\text { Liés à la régulation de la } \\
\text { qualité de l'eau }\end{array}$} & \multirow{2}{*}{ Bon état (DCE) } \\
\hline & Environnement olfactif & & \\
\hline & Tourisme et loisirs & $\begin{array}{l}\text { Qualité de l'eau pour la } \\
\text { baignade, aspects esthétiques, } \\
\text { olfactifs, pêche de loisirs... }\end{array}$ & $\begin{array}{l}\text { Bon état (DCE), } \\
\text { SEQ loisirs }\end{array}$ \\
\hline $\begin{array}{lr}\text { Les } & \text { zones } \\
\text { industrielles } & \text { et } \\
\text { commerciales } & \end{array}$ & $\begin{array}{l}\text { Approvisionnement en } \\
\text { eau }\end{array}$ & $\begin{array}{l}\text { Qualité de l'eau pour les } \\
\text { différents } \\
\text { d'approvisionnement } \text { types } \\
\text { l'industrie : eau potable et eau } \\
\text { de process }\end{array}$ & $\begin{array}{l}\text { Bon état (DCE), } \\
\text { SEQ production } \\
\text { d'eau potable }\end{array}$ \\
\hline $\begin{array}{l}\text { L'Estuaire fluvial } \\
\text { Garonne aval }\end{array}$ & $\begin{array}{l}\text { Régulation de la qualité } \\
\text { de l'eau }\end{array}$ & $\begin{array}{l}\text { De l'eau en qualité et en } \\
\text { quantité nécessaire pour } \\
\text { assurer les phénomènes } \\
\text { d'autoépuration }\end{array}$ & $\begin{array}{l}\text { Bon état } \\
\text { écologique } \\
\text { (DCE), NQE }\end{array}$ \\
\hline
\end{tabular}

\subsection{2. Étape 4.2 Élaboration de seuils de rejets et de rendements épuratoires pour le traitement des eaux usées du projet}

Pour la Jalle de Blanquefort, les critères à respecter issus de l'étude approfondie des normes et des textes réglementaires sont répertoriés dans le tableau 9.

Les concentrations retenues dans la première colonne du tableau 9 correspondent donc à une composition du milieu qui permettrait de maintenir les différents services produits par la Jalle de Blanquefort qui sont susceptibles de pouvoir subir des dommages. De plus, ce tableau 9 présente les résultats des concentrations maximales admissibles calculés par des effets de dilution.

Tableau 9 : Calcul des concentrations maximales admissibles par la Jalle de Blanquefort véhiculées par un débit égal au débit de rejet ( - » = donnée non disponible)

\begin{tabular}{|c|c|c|c|}
\hline Paramètres & $\begin{array}{l}\text { Concentrations } \\
\text { maximales } \text { retenues } \\
\text { pour la Jalle de } \\
\text { Blanquefort }(2011)\end{array}$ & $\begin{array}{l}\text { Concentrations } \\
\text { dans la Jalle de } \\
\text { Blanquefort (2012) }\end{array}$ & $\begin{array}{l}\text { Concentrations } \\
\text { maximales admissibles } \\
\text { dans la Jalle de } \\
\text { Blanquefort pour un rejet } \\
\text { de débit }\end{array}$ \\
\hline Débit $\left(\mathrm{m}^{3} / \mathrm{j}\right)$ & & 5270 & 273 \\
\hline DCO (mg/L) & 6 & - & \\
\hline
\end{tabular}




\begin{tabular}{|c|c|c|c|}
\hline $\mathrm{DBO}_{5}(\mathrm{mg} / \mathrm{L})$ & 3 & 5 & 23 \\
\hline $\mathrm{NKJ}(\mathrm{mg} / \mathrm{L})$ & 1 & - & $<19$ \\
\hline $\begin{array}{l}\text { Phosphore total } \\
\text { (PT) }(\mathrm{mg} / \mathrm{L})\end{array}$ & 0.01 & 0.37 & -4 \\
\hline $\operatorname{MES}(\mathrm{mg} / \mathrm{L})$ & 2 & - & $<39$ \\
\hline Cuivre $(\mu \mathrm{g} / \mathrm{L})$ & 1.4 & 0.17 & 23.7 \\
\hline $\begin{array}{l}\text { Zinc (dureté } \\
\text { moyenne) ( } \mu g / \\
\text { L) }\end{array}$ & 4 & 5 & $\mid-19.3$ \\
\hline $\begin{array}{l}\text { Chloroforme } \\
(\mu \mathrm{g} / \mathrm{L})\end{array}$ & 2.5 & 0.35 & 41.5 \\
\hline Chrome $(\mu \mathrm{g} / \mathrm{L})$ & 3.4 & 0.35 & 58.9 \\
\hline $\begin{array}{l}\text { Nonylphénols } \\
(\mu \mathrm{g} / \mathrm{L})\end{array}$ & 0.3 & 0.13 & 3.28 \\
\hline Nickel $(\mu \mathrm{g} / \mathrm{L})$ & 20 & 1 & 366.8 \\
\hline Plomb $(\mu \mathrm{g} / \mathrm{L})$ & 7.2 & 0.02 & 138.6 \\
\hline
\end{tabular}

La même analyse est menée pour l'estuaire fluvial Garonne aval et ne sera pas détaillé ici.

Finalement, le tableau 10 présente les concentrations maximales retenues pour un rejet en milieu naturel en fonction des contraintes les plus importantes entre les contraintes liées au milieu et les contraintes règlementaires applicables au site. Ce tableau présente également les rendements minimums des filières de traitement en fonction de la composition actuelle de l'effluent de l'entreprise. Nous remarquons que les valeurs les plus contraignantes sont celles liées au milieu naturel et au maintien des services écosystémiques produits par la Jalle de Blanquefort et l'estuaire fluvial Garonne aval.

Ces résultats permettent ainsi d'apporter des éléments à la rédaction du cahier des charges pour dimensionner les installations. 
Tableau 10 : Concentrations maximales acceptables pour le rejet en milieu naturel des effluents aqueux de l'entreprise Castel Frères (site de Blanquefort) en fonction des critères de maintien des services écosystémiques produits par l'aire d'étude et de la règlementation en vigueur pour le secteur d'activité - calcul des rendements nécessaires pour les procédés de traitement à mettre en place

\begin{tabular}{|c|c|c|c|c|c|c|}
\hline & $\begin{array}{l}\text { Seuils de } \\
\text { concentration } \\
\text { maximum } \\
\text { acceptables } \\
\text { par le milieu }\end{array}$ & $\begin{array}{l}\text { Valeurs } \\
\text { limites } \\
\text { d'émission } \\
\text { générales } \\
\text { applicables } \\
\text { (Arrêté du } \\
02 / 02 / 98 \text { ) }\end{array}$ & $\begin{array}{l}\text { Valeurs } \\
\text { limites } \\
\text { d'émission } \\
\text { définies } \\
\text { par } \\
\text { l'arrêté } \\
\text { sectoriel } \\
\text { du } \\
19 / 03 / 99\end{array}$ & $\begin{array}{l}\text { Seuils de } \\
\text { concentrations } \\
\text { retenus pour } \\
\text { le rejet de } \\
\text { l'entreprise de } \\
\text { débit } Q_{R}\end{array}$ & $\begin{array}{l}\text { Effluent } \\
\text { brut } \\
\text { entreprise } \\
\text { (moyenne } \\
\text { annuelle) }\end{array}$ & $\begin{array}{l}\text { Rendement } \\
\text { minimum } \\
\text { des filières } \\
\text { de } \\
\text { traitement } \\
(\%)\end{array}$ \\
\hline Débit $\left(\mathrm{m}^{3} / \mathrm{j}\right)$ & $273\left(\mathrm{Q}_{\mathrm{R}}\right)$ & - & & & 273 & - \\
\hline $\mathrm{DBO}_{5}(\mathrm{mg} / \mathrm{L})$ & 23 & 30 & 30 & 23 & 1935 & 98.8 \\
\hline NKJ (mg/L) & 19 & - & - & 19 & 20 & 5 \\
\hline $\begin{array}{l}\text { Phosphore } \\
\text { total (PT) } \\
(\mathrm{mg} / \mathrm{L})\end{array}$ & 0.01 & - & - & 0.01 & 11 & 99.9 \\
\hline MES (mg/L) & 2 & 35 & 35 & 2 & 171 & 98.8 \\
\hline Cuivre ( $\mu \mathrm{g} / \mathrm{L})$ & 23.7 & 500 & - & 23 & 40.7 & 44 \\
\hline $\begin{array}{l}\text { Zinc (dureté } \\
\text { moyenne) } \\
(\mu \mathrm{g} / \mathrm{L})\end{array}$ & 4 & 200 & - & 4 & 228 & 98.2 \\
\hline $\begin{array}{l}\text { Chloroforme } \\
(\mu \mathrm{g} / \mathrm{L})\end{array}$ & 41.5 & - & - & 41 & 34.11 & - \\
\hline $\begin{array}{l}\text { Chrome ( } \mu \mathrm{g} / \\
\mathrm{L})\end{array}$ & 58.9 & 500 & - & 58 & 13.5 & - \\
\hline $\begin{array}{l}\text { Nonylphénols } \\
(\mu \mathrm{g} / \mathrm{L})\end{array}$ & 3.28 & - & - & 3.2 & 2.06 & - \\
\hline Nickel ( $\mu \mathrm{g} / \mathrm{L})$ & 366.8 & 500 & - & 366 & 11.1 & - \\
\hline Plomb $(\mu \mathrm{g} / \mathrm{L})$ & 138.6 & 500 & - & 138 & 5.95 & - \\
\hline
\end{tabular}




\section{Discussion}

\subsection{Déséquilibres de pouvoir entre acteurs}

81 Une des conclusions de la partie 1 est la mise en évidence d'un déséquilibre entre les acteurs dans le processus décisionnel. Nous avions identifié deux acteurs forts (l'industriel et le préfet de département délivrant l'autorisation) et des acteurs faibles, notamment les acteurs représentant les milieux naturels. La méthode proposée permet d'identifier à un stade précoce du projet l'ensemble des parties prenantes potentiellement concernées par les effets du rejet afin de mieux prendre en compte leurs besoins vis-à-vis des services écosystémiques produits par le milieu récepteur. Ces besoins sont ensuite pris en compte pour l'élaboration de seuils de rejet correspondant aux besoins les plus contraignants. Ainsi, un certain équilibre est rétabli entre acteurs forts et acteurs faibles grâce à une prise en compte directe des milieux naturels et parties prenantes associées par le porteur de projet.

\subsection{Des acteurs aux référentiels différents}

82 L'application de la méthode élaborée a mis en exergue la divergence entre les paramètres de suivi des masses d'eau et les paramètres de contrôle de l'entreprise Castel, notamment sur les paramètres « classiques ». En effet, pour l'entreprise, des paramètres tels que la $\mathrm{DCO}$, la $\mathrm{DBO}_{5}$ et l'azote Kjeldhal sont mesurés dans le cadre de son arrêté d'autorisation, alors que pour la masse d'eau réceptrice du rejet, les paramètres de suivi sont le carbone organique, la $\mathrm{DBO}_{5}$, l'ammonium, les nitrites et les nitrates.

83 Ce point particulier met en évidence le manque de lien entre la règlementation environnementale «industrielle» (ICPE, IED...) et celle sur les milieux (DCE), en particulier dans la partie règlementaire. Ceci entraine donc certaines difficultés dans la mise en application.

\subsection{Utilisation des services écosystémiques.}

84 La méthode élaborée est basée sur les besoins de l'être humain vis-à-vis des services écosystémiques produits par les milieux. Ce sont donc les besoins de l'humain, en tant que clients secondaires, ou parties prenantes, qui sont pris en considération. Cette approche reste donc très anthropocentrée.

Les «matrices portraits" élaborées permettent d'identifier des dommages potentiellement générés par le projet, c'est-à-dire les services écosystémiques pouvant être impactés. Suivant les services et les composantes concernés, les cibles potentielles sont identifiées comme parties prenantes. Les besoins des cibles permettent d'établir des seuils en termes de concentrations de différents paramètres pour la composante concernée. Ces seuils identifiés pour le milieu permettent de calculer une capacité d'acceptation de rejet. Ainsi, la méthodologie proposée permet à l'entreprise, «à priori ", d'aboutir à des caractéristiques de rejet qui soit compatible avec les besoins des parties prenantes vis-à-vis des services produits par les milieux. 


\section{Conclusion et perspectives}

Dans le contexte actuel où malgré une règlementation foisonnante en matière de protection de l'environnement, l'Europe fait face à des difficultés dans l'atteinte des objectifs de qualité de ses milieux aquatiques. Cet article présente une réflexion sur l'élaboration de seuils de rejets aqueux écocompatibles. Ces seuils de rejet sont obtenus en combinant l'approche qualité (ISO 9000) et celle des services écosystémiques dans une démarche en quatre étapes.

Les travaux qui ont été menés admettent quelques limites. Il s'agit notamment de sa validation à travers une étude de cas unique, une approche qui reste anthropocentrée, et des seuils de rejet proposés qui correspondent à un rejet «seul » dans le milieu, c'est-àdire que les capacités d'acceptation du milieu sont utilisées en totalité, ne laissant pas de " place » à un autre rejet éventuel.

Ces travaux amènent également à plusieurs perspectives. La première est l'intégration d'outils de modélisation de bassins versants à la méthode afin de pouvoir prendre en compte les phénomènes d'autoépuration des milieux ainsi que les impacts cumulatifs avec d'autres rejets/prélèvements, confluences, etc. dans l'élaboration de seuils de rejets. La seconde est l'utilisation de l'outil dans d'autres configurations que celle de l'étude de cas où l'industrie est déjà en exploitation et où de nombreuses données sont donc disponibles.

\section{BIBLIOGRAPHY}

BAKER, J., SHEATE, W.R., PHILLIPS, P. et EALES, R. 2012, « Ecosystem services in environmental assessment - Help or hindrance? », Environmental Impact Assessment Review, vol. 40, p. 3-13. BENEDINI, M. (2011). « Water quality models for rivers and streams. State of the art and future perspectives », European water, vol. 34, p. 17-40.

BOSSARD, M., FERANEC, J. et OTAHEL, J. (2000), Corine Land Cover technical guide - addendum 2000, European Environment Agency, Copenhague, 105 p.

BURKHARD, B., KROLL, F. MÜLLER, F. et WINDHORST, W. (2009). « Landscapes' capacities to provide ecosystem services-a concept for land-cover based assessments ", Landscape online, vol. 15, p. 1-22.

CANNON, W.B. (1963), The wisdom of the body. W. W. Norton \& company, 340 p.

CERCEAU, J. (2013) L'écologie industrielle comme processus de construction territoriale : application aux espaces portuaires, thèse de doctorat, Mines Saint-Etienne, 356 p.

CNRS (2014), « Dégradations - La pollution par les matières organique et la pollution microbiologique », Le CNRS en ligne, http://www.cnrs.fr/cw/dossiers/doseau/decouv/ degradation/05_pollution.htm (consulté le 19 mars 2015). 
COUVET D., X. ARNAULD DE SARTRE, E. BALIAN ET TICHIT. M. (2016) « Services écosystémiques : des compromis aux synergies ", in RochE, P., I. GEIJZENDORFFER, H. LEVREL et MARIS, V. (Ed.), Valeurs de la biodiversité et services écosystémiques, Perspectives interdisciplinaires, Editions Quae, p. 143-160.

cox, B. (2003) « A review of currently available in-stream water-quality models and their applicability for simulating dissolved oxygen in lowland rivers ", Science of The Total Environment, vol. 314-316, p. 335-377.

EEA (2015). « The European environment - state and outlook 2015: synthesis report », European Environment Agency, http://www.eea.europa.eu/soer (consulté le 23 mars 2017).

FISHER B., R. COSTANZA, R.K.TURNER et P. MORLING (2009) « Defining and classifying ecosystem services for decisions making ", Ecological economics, vol. 68, p. 643-653.

GRAZILHON. S. (2015). Méthode d'aide à la décision pour la qualité des effluents industriels. Une approche territoriale orientée sur les besoins vis-à-vis des services écosystémiques produits par les milieux récepteurs, Thèse de l'École Nationale Supérieure des Mines de Saint-Etienne, 211 p.

IGN (2015). Géoportail, portail des territoires et des citoyens, http://www.geoportail.gouv.fr (consulté le 17 mars 2015).

ISO (2005). « ISO 9000 :2005 Systèmes de management de la qualité - Principes essentiels et vocabulaire ", Organisation internationale de normalisation, https://www.iso.org/fr/ standard/42180.html (consulté le 23 mars 2017).

KNOWLES, G. et WAKEFORD, A.C. (1978). « A mathematical deterministic river-quality model. Part 1: Formulation and description ", Water research, vol. 12, no 12, p. 1149-1151.

LAROUSSE (2014) « Qualité », Larousse, http://www.larousse.fr/encyclopedie/rechercher?q=qualit\% C3\%A9 (consulté le 23 mars 2017)

LE GOFF, F., et BONNOMET, V. (2004). Devenir et comportement des métaux dans l'eau : biodisponibilité et modèles BLM, Rapport technique INERIS, $87 \mathrm{p}$.

MANNINA, G. et VIVIANI, G. (2010). « River water quality assessment : A hydrodynamic water quality model for propagation of pollutants ", 7e Conférence internationale sur les techniques et stratégies durables, Lyon, France, 28-30 juin 2010, p. 1-10.

MASLOW, A.H. (1943). «A theory of human motivation », Psychological Review, vol. 50, p. 370-396.

MEA (2005). Ecosystems and human well-being - Synthesis, Millennium Ecosystem Assessment, Island Press edn, Washington, DC, $155 \mathrm{p}$.

MEDDE (2010) Référentiel pour la constitution d'un Dossier de Demande d'Autorisation d'Exploiter (DDAE) des installations classées en Nord-Pas-de-Calais, Ministère de l'Écologie, de l'Énergie, du Développement durable et de l'Aménagement du territoire, $68 \mathrm{p}$.

MICHEL, P. (2001). L'étude d'impact sur l'environnement, Ministère de l'Aménagement du territoire et de l'Environnement, $157 \mathrm{p}$.

NOLTEMEYER, A., BUSH, K., PATTON, J. et BERGEN, D. (2012). « The relationship among deficiency needs and growth needs: An empirical investigation of Maslow's theory ", Children and Youth Services Review, vol. 34, no 9, p. 1862-1867.

ONEMA/OIEAU (2015) L'état des eaux de surface et des eaux souterraines, États des lieux - Agences de l'eau, DREAL délégations de bassin - 2013, no 12, 12 p.

OREE (2009). Mettre en ceuvre une démarche d'écologie industrielle sur un parc d'activités, la collection Orée, $252 \mathrm{p}$. 
PARAN, F., GRAILLOT, D. et DECHOMET, R. (2010). « Caractérisation spatio-temporelle des échanges nappes/rivière à l'échelle de deux grands fleuves français : application aux secteurs de DonzèreMondragon (Rhône) et Ecozone du Forez (Loire) », Colloque Eau, Déchets et Développement Durable, Alexandrie, Égypte, 31 mars 2010.

PARLEMENT EUROPÉEN ET CONSEIL DE L'UNION EUROPÉENNE (2010). Directive n 2010/75/UE du 24/11/2010 relative aux émissions industrielles (prévention et réduction intégrées de la pollution) (Refonte), Directive edn, Union Européenne.

RISHI, P., MOGHE, S. et UPADHYAY, B.K. (2008). « Analysis of hierarchy of needs and motivational strategies for eco-development planning in two national parks of India », Resources, Conservation and Recycling, vol. 52, no 5, p. 707-718.

PYKH, Y.A. et MALKINA-PYKH, I.G. (1997). « POLMOD.PEST-the model of pesticides dynamics in the elementary ecosystems », Ecological Modelling, vol. 98, no. 2-3, p. 215-236.

SAlla, M.R., ARQUiola, J.P., LERMA ElVIRA, N., ALAMY FILHO, J. E., PEREIRA, C.E. et SILVA COSTA, E. (2015). "Application of the EVALHID tool for parameter calibration and flow simulation in the upper course of Araguari river, Minas Gerais ", Revista brasileira de Recursos Hidricos, vol. 20, p. 276-285.

SALLA, M.R., FERNANDES, L.E., PEREIRA, C.E., CORREIA DA SILVA JHUNIOR, H. et DE LIMA, G. (2016).

« Importance of calibration for mathematical modeling of self-purification of lotic environments ", Acta Limnologica Brasiliensia, vol. 28, p. 1-18.

SEBASTIEN, L. (2011). Quand les acteurs faibles et absents s'immiscent dans la négociation environnementale, Territoire en mouvement Revue de géographie et aménagement, vol. 11, 66-81.

TABUCHI, J., L. BENARD, M. GousAilles, S. LEgRUel, R. NEDELEC, B. BLANCHET et M. POULIN (2012). « Apports de la modélisation de la qualité de la Seine à la définition des projets de stations d'épuration et à la gestion en temps réel du système d'assainissement », IS.RIVERS 2012, A3 Metropole/large cities, France, p. 1-3.

VAGNETTI, R., MIANA, P., FABRIS, M. et PAVONI, B. (2003). « Self-purification ability of a resurgence stream », Chemosphere, vol.52, no 10, p. 1781-1795.

WALLIS, C., BLANCHER, P., SEON-MASSIN, N., MARTINI, F. et SCHOUPPE, M. (2011), Mise en œuvre de la Directive Cadre sur l'Eau - Quand les services écosystémiques entrent en jeu, Les Rencontre de l'ONEMA, Bruxelles, $107 \mathrm{p}$.

WHITEHEAD, P.G. et R.J. WILLIAMS ET D.R. LEWIS (1997). « Quality simulation along river systems (QUASAR): model theory and development ", Science of The Total Environment, vol. 194-195, no 0, p. 447-456.

\section{ABSTRACTS}

This article concern the possibilities of using quality management principles prescribed by the ISO 9000 for industrial wastewater management, with potential customers ecosystem receptors. The consideration of ecosystems as customers nevertheless raises sensitive issues such as the assessment of their needs. The territory is considered in terms of ecosystem services to identify human targets able to express their needs concerning their environment. These needs are then converted, according to local and regulatory data, in qualitative requirements for liquid emissions. This approach was materialized as a tool for industrials 
Cet article s'intéresse à l'utilisation des principes de gestion de la qualité prescrits par l'ISO 9000, à la gestion des effluents industriels, avec comme clients potentiels notamment les écosystèmes récepteurs. La prise en compte des écosystèmes comme clients soulève la question de l'évaluation de leurs besoins. Le territoire est alors considéré par le biais des services écosystémiques afin d'identifier des cibles humaines aptes à exprimer leurs besoins afin d'en tirer des exigences vis-àvis du milieu qui sont ensuite converties en exigences qualitatives vis-à-vis des rejets. Cette démarche a été matérialisée sous la forme d'un outil destiné aux industriels.

INDEX

Mots-clés: services écosystémiques, qualité des effluents industriels, acteurs, milieux récepteurs Keywords: ecosystem services, industrial effluents quality, actors, receiving media

\section{AUTHORS}

\section{VALÉRIE LAFOREST}

Univ Lyon, École Nationale Supérieure des Mines de Saint-Étienne, CNRS, UMR 5600 EVS, F-42023

SOPHIE GRAZILHON

Univ Lyon, École Nationale Supérieure des Mines de Saint-Étienne, CNRS, UMR 5600 EVS, F-42023

\section{ERIC PIATYSZEK}

Univ Lyon, École Nationale Supérieure des Mines de Saint-Étienne, CNRS, UMR 5600 EVS, F-42023 